\title{
PARP inhibition in leukocytes diminishes inflammation via effects on integrins/ cytoskeleton and protects the blood-brain barrier
}

Slava Rom ${ }^{1,2^{*}}$, Viviana Zuluaga-Ramirez ${ }^{1}$, Nancy L. Reichenbach ${ }^{1}$, Holly Dykstra ${ }^{1}$, Sachin Gajghate ${ }^{1}$, Pal Pacher ${ }^{3}$ and Yuri Persidsky ${ }^{1,2^{*}}$

\begin{abstract}
Background: Blood-brain barrier (BBB) dysfunction/disruption followed by leukocyte infiltration into the brain causes neuroinflammation and contributes to morbidity in multiple sclerosis, encephalitis, traumatic brain injury, and stroke. The identification of pathways that decreases the inflammatory potential of leukocytes would prevent such injury. Poly(ADP-ribose) polymerase 1 (PARP) controls various genes via its interaction with myriad transcription factors. Selective PARP inhibitors have appeared lately as potent anti-inflammatory tools. Their effects are outside the recognized PARP functions in DNA repair and transcriptional regulation. In this study, we explored the idea that selective inhibition of PARP in leukocytes would diminish their engagement of the brain endothelium.

Methods: Cerebral vascular changes and leukocyte-endothelium interactions were surveyed by intravital videomicroscopy utilizing a novel in vivo model of localized aseptic meningitis when TNFa was introduced intracerebrally in wild-type $\left(\mathrm{PARP}^{+/+}\right)$and PARP-deficient $\left(\mathrm{PARP}^{-/}\right)$mice. The effects of selective PARP inhibition on primary human monocytes ability to adhere to or migrate across the BBB were also tested in vitro, employing primary human brain microvascular endothelial cells (BMVEC) as an in vitro model of the BBB.

Results: PARP suppression in monocytes diminished their adhesion to and migration across BBB in vitro models and prevented barrier injury. In monocytes, PARP inactivation decreased conformational activation of integrins that plays a key role in their tissue infiltration. Such changes were mediated by suppression of activation of small Rho GTPases and cytoskeletal rearrangements in monocytes. In vitro observations were confirmed in vivo showing diminished leukocyte-endothelial interaction after selective PARP suppression in leukocytes accompanied by BBB protection. PARP knockout animals demonstrated a substantial diminution of inflammatory responses in brain microvasculature and a decrease in BBB permeability.

Conclusions: These results suggest PARP inhibition in leukocytes as a novel approach to BBB protection in the setting of endothelial dysfunction caused by inflammation-induced leukocyte engagement.
\end{abstract}

Keywords: Leukocyte-endothelial interaction, VLA-4, LFA-1, Actin cytoskeleton, PARP-1

\footnotetext{
*Correspondence: srom@temple.edu; yuri.persidsky@tuhs.temple.edu

'Department of Pathology and Laboratory Medicine, Temple University,

Philadelphia, PA 19140, USA

Full list of author information is available at the end of the article
} 


\section{Background}

Leukocyte adhesion and migration are mediated by integrins, adhesion receptors expressed on diverse cell types participating in cell-to-cell interactions [1-4]. Integrins facilitate bi-directional signaling. Outside-in transmission involves integrin ligation resulting in stimulation of several signaling pathways. Ligation of other receptors can lead to transmission of an inside-out signal towards the integrin [5]. This signaling usually results in integrin's conformational modifications (bending/unbending) leading to a rapid rise or decline in integrin-ligand binding affinity and changes in integrin lateral mobility, which directly regulate cell adhesion potential $[4,6]$ and is critical for immune system responses [6]. Most circulating leukocytes display a non-adhesive phenotype, having integrins in a resting/inactive state. Leukocytes may roll on endothelial cells, arrest, firmly adhere, and transmigrate across the endothelial barrier. In this study, we focused on two major leukocyte integrins $[C D 49 d / C D 29$, very late antigen-4 (VLA-4), alpha4 beta1 integrin], and [CD11a/CD18, lymphocyte function-associated antigen-1 (LFA-1), alpha L beta2 integrin]. VLA-4 and LFA-1 directly mediate cell arrest under flow conditions, where firm adhesion is mediated by activated (high-affinity, unbent) integrins. The activation of integrins, adhesion and migration processes involve a chain of actin cytoskeleton rearrangements and activation of small Rho GTPases [6-9].

In the search for anti-inflammatory/neuroprotective compounds, we focused on poly(ADP-ribose) polymerase1 (PARP) inhibitors (further referred to as PARPi) that are currently used in the treatment of cancer [10]. PARP is responsible for synthesis and transfer of ADP-ribose polymers to target proteins, regulation of DNA repair, and genomic integrity maintenance [11]. PARP hyperactivity has been found in a number of central nervous system (CNS) disorders (including ischemia, neurodegeneration, and neuroinflammation) [12]. PARP inhibition in macrophages/microglia diminished production of inflammatory mediators [11], decreased the number of $\mathrm{T}$ cell subsets, while increasing the amount of anti-inflammatory cytokines and regulatory, suppressive T lymphocytes [13]. The first PARPi were discovered in 1980 [14, 15]. All known PARPis are nicotinamide mimetics that bind in the NAD+ binding pocket of the catalytic domain, with the exception of indole and coumarin derivatives that bind in the zincfinger domains in PARP [14-16]. In the current study, we used PARPis belonging to the nicotinamide mimetic group; two of them (olaparib and talazoparib) are currently in clinical trials for treatment of several malignancies (phase II and III), offering rapid translation to therapy of immune/inflammatory disorders [10]. PARPi have been shown to be anti-inflammatory and tissue-protective in animal models of traumatic brain injury (TBI) [17], multiple sclerosis (MS) [18], meningitis [19], arthritis [20], and lung, liver, and kidney injury [21].

Inflammation plays a significant role in neuronal dysfunction and neurocognitive impairment [22]. Neuroinflammatory responses are characterized by activation of resident macrophages and microglia that release cytokines, chemokines, and proteolytic enzymes negatively affecting neural cells. Inflammatory molecules can stimulate the brain endothelium from the abluminal (brain) side and increase expression of adhesion molecules on the luminal side [23], leading to enhanced leukocyte engagement of the brain endothelium and subsequent migration across the blood-brain barrier (BBB) into the neuropil, further aggravating neuroinflammation [24]. In systemic inflammation (e.g., sepsis) [25], there is an enhanced adhesion of leukocytes in different organs (including CNS) as well as cytokine production that result in increased in $\mathrm{BBB}$ permeability. During stroke, in response tissue injury/local cytokine/ chemokine production, neutrophils infiltrate ischemic brain (in $30 \mathrm{~min}$ to a few hours), while monocytes/macrophages migrate within a day or two, and T cells follow on day 3 to 7 after an ischemic event [26, 27]. Lymphocyte/monocyte infiltration into the CNS is a signature event in multiple sclerosis (MS), an inflammatory condition of the CNS. It remains enigmatic whether BBB dysfunction leads to immune cell infiltration or it is the result of perivascular leukocyte accumulation, but leukocyte migration definitely modifies BBB permeability. Leukocytes in MS express inflammatory cytokines, reactive oxygen species (ROS), and enzymes that can augment their egress into the CNS by influencing $\mathrm{BBB}$ function, either directly or indirectly [28, 29]. This process is accompanied by $\mathrm{BBB}$ injury and increased permeability to blood components toxic to neurons [30]. Therefore, approaches diminishing leukocyte interactions with brain endothelial cells may prevent BBB damage and attenuate neuroinflammation, resulting in neuroprotection [31]. Inhibiting leukocyte migration during the first stages of inflammation (in sepsis, MS, or ischemia/reperfusion) may have positive effects by reducing tissue injury, but on the other hand, prolonged inhibition might have negative effects in terms of uncontrolled infections or prevention of immune cells from initiation of regenerative activities, since inflammation is a vital part of the physiological reaction towards tissue repair and plasticity [32-34]. It is essential to look for anti-inflammatory treatments and to study their mechanisms of action to be able to fine tune therapeutic approaches.

Our prior work revealed that PARP inhibition in primary human brain microvascular endothelial cells (BMVEC) decreased monocyte adhesion and migration across BMVEC monolayers and attenuated expression of 
adhesion molecules. PARP suppression in BMVEC attenuated activity of small GTPases, dampened expression and secretion of pro-inflammatory factors [35]. Utilizing an in vivo aseptic meningitis/encephalitis model, we demonstrated diminished adhesion and migration of leukocytes across the $\mathrm{BBB}$, confirming our in vitro observations.

While we could selectively inhibit PARP in vitro in BMVEC, it was impossible to distinguish whether decreased adhesion/migration in vivo resulted from PARP inhibition in endothelium or from PARP suppression in leukocytes as well. To challenge this issue, we used adoptive transfer of leukocytes and intravital videomicroscopy (IVM) and found that leukocytes from PARP1 ${ }^{-/}$mice (PARPko) displayed reduced adhesion and migration across cortical vessel endothelium vs. their wildtype $\mathrm{PARP}^{+/+}$(WT) counterparts. To address putative anti-inflammatory effects of PARP inhibition in leukocytes, we pretreated human monocytes with PARPi and showed their decreased adhesion/migration across $\mathrm{BBB}$ models, paralleling attenuation of active integrin expression, cytoskeletal alterations, and GTPase activity. Genetic or chemical inhibition of PARP in vivo prevented their migration across the $\mathrm{BBB}$ and diminished barrier permeability.

\section{Methods}

\section{Reagents and cell culture}

The selective PARPi used were 5-aminoisoquinolinone (AIQ; Enzo Life Sciences, Farmingdale, NY); olaparib and talazoparib (Selleck Chemicals, Houston, TX), 5' -deoxy5' -[4-[2-[(2, 3-dihydro-1-oxo-1H-isoindol-4-yl) amino]-2oxoethyl]-1-piperazinyl]-5' -oxoadenosine dihydrochloride (EB47; Tocris Bioscience, Minneapolis, MN). The RhoA inhibitor, CT04, and RhoA/Rac1 activator, CN04, were from Cytoskeleton (Denver, CO). The Rac1 inhibitor, NSC23766, was from EMD Millipore (Billerica, MA). Lipopolysaccharide (LPS) from Escherichia coli 0111:B4 and Rhodamine 6G were from Sigma-Aldrich (St. Louis, MO). Recombinant human tumor necrosis factor- $\alpha$ (TNF $\alpha$ ) and human monocyte chemotactic protein type-1 (MCP1/CCL2) were from R\&D Systems (Minneapolis, $\mathrm{MN})$. Phorbol 12-myristate 13-acetate (PMA) was from Cayman Chemical (Ann Arbor, MI).

Primary human monocytes from HIV-1/hepatitis B seronegative donors were obtained from the University of Nebraska Medical Center [36]. Primary brain microvascular endothelial cells (BMVEC) were provided by Dr. M. Witte (University of Arizona, Tucson, AZ), isolated from the temporal cortex obtained during surgical removal of eleptogenic foci in adult patients [37] and maintained in culture as described [9]. Cell culture reagents were from Life Technologies (Carlsbad, CA).
Monocytes were pretreated with PARPi (AIQ $1 \mu \mathrm{M}$, olaparib, EB47, $10 \mu \mathrm{M}$; talazoparib, 10,25 , or $10 \mathrm{nM}$ ) [35, 38, 39] or RhoA/Rac1 activator/inhibitor (CN04, $1 \mu \mathrm{g} / \mathrm{ml}$; CT04, $1 \mu \mathrm{g} / \mathrm{ml}$; NSC23766, $75 \mu \mathrm{mol} / \mathrm{l}$ ) for $30 \mathrm{~min}$ unless otherwise noted and did not affect cell viability [39]. Dose-response results are shown in Additional file 1: Figure S1. In all experiments, the designation non-treated (NT) indicates that medium only was added to the cells.

\section{Animals}

C57BL/6 mice (8-week-old male) were from the Jackson Laboratory (Bar Harbor, MI). PARPko mice $\left(\mathrm{PARP}^{-/-}\right.$) were generated on C57BL/6J background [40] provided by Dr. P. Pacher (NIAAA). To achieve statistical significance in each experiment, mice were divided into groups of four to six animals (exact numbers for each experiment are indicated in figure legends).

All in vivo experiments were approved by the Temple University Institutional Animal Care and Use Committee in accordance with guidelines based on the National Institutes of Health (NIH) guide for care and use of laboratory animals and ARRIVE (Animal Research: Reporting In Vivo Experiments) guidelines (www.nc3rs.org.uk/arriveguidelines).

\section{IVM and ex vivo treatment and labeling of leukocytes}

Prior to IVM, mice underwent craniotomy and cranial window implantation as described [41]. Prior to IVM, mice were treated with LPS $(6 \mathrm{mg} / \mathrm{kg})$ i.v. or TNF $\alpha$ $(0.5 \mu \mathrm{g} /$ mouse) by IC injection $[9,35,42,43]$. Two hours post-injection of TNF $\alpha$, leukocytes were labeled in vivo with Vybrant ${ }^{\bullet}$ DiI Cell-Labeling Solution (DiI) (Life Technologies, Carlsbad, CA) introduced i.v. Leukocyte adhesion was detected in cerebral vessels through the cranial window using a SteREO Discovery V20 epifluorescence microscope (Carl Zeiss Microimaging Inc., Thornwood, NY) equipped with a AxioCam MR digital camera as previously described [35, 44]. A 30-s video (time-series image set between 16 and 20 frames/s) was captured using the digital high-speed recorder. Adherent leukocytes were defined as the number of leukocytes firmly attached to the endothelium that did not change location during the observation period, scored as the number of cells per $\mathrm{mm}^{2}$ of the vascular surface area, calculated from the diameter and length of the vessel segment under observation. Imaris 8.3 software (Bitplane AG, Zurich, Switzerland) was used to count adherent leukocytes. Transmigrated leukocytes were enumerated $24 \mathrm{~h}$ later in an area covering a distance of $100 \mu \mathrm{m}$ from the pial and parenchymal vessel wall by epifluorescent IVM. The number of extravasated leukocytes was counted and normalized to area, using ImageJ software (National Institutes of Health, Bethesda, MD) [35]. 
Leukocytes were isolated from five donor mice PBMC with red blood cell lysis buffer (eBioscience, Inc., San Diego, CA). Leukocytes $\left(2 \times 10^{6}\right)$ were ex vivo treated with PARPi for $30 \mathrm{~min}$, washed with PBS, and labeled with calcein-AM (1 $\mu \mathrm{M}$, Life Technologies) as described [9]. Concomitantly, recipient mice were treated with LPS or TNF $\alpha$ as described above for $4 \mathrm{~h}$, and mice were anesthetized, injected intra-orbitally with Rhodamine 6G to label autologous leukocytes, imaged by IVM, and then injected with calcein-AM-labeled leukocytes. Leukocytes were visualized by fluorescent light (495 or $601 \mathrm{~nm}$ excitation for calcein-AM or Rhodamine 6G, respectively). Ex vivo treatment of leukocytes did not change the population profile (Additional file 1: Figure S2B).

\section{In vivo permeability assay}

Changes in BBB permeability were assessed using the fluorescent tracer, sodium-fluorescein $(\mathrm{Na}-\mathrm{F})$ as described $[35,41,43]$. Briefly, to prevent blood clotting in the vessels, animals were injected i.p. with heparin (20 U) followed i.v injection of $50 \mu \mathrm{l}$ of $2 \% \mathrm{Na}-\mathrm{F}$ in PBS. The tracer was allowed to circulate for $30 \mathrm{~min}$. The mice were anesthetized and then transcardially perfused with PBS until colorless perfusion was visualized. The animals were then decapitated and the brains were quickly isolated. After removal of the meninges, cerebellum, and brain stem, the tissue was weighed and homogenized in 10 times volume of $50 \%$ trichloroacetic acid. The homogenate was centrifuged for $10 \mathrm{~min}$ at $13,000 \times \mathrm{g}$, and the supernatant was neutralized with $5 \mathrm{M} \mathrm{NaOH}$ (1:0.8). The amount of Na-F was measured using a Synergy 2 plate reader (BioTek, Winooski, VT). Fluorescent dye content was calculated using external standards; data are expressed as amount of tracer per milligrams of tissue.

\section{PARP activity assay}

To measure PARP activity, leukocytes were treated with/ out PARPi for $30 \mathrm{~min}$ and then lysed and subjected to ELISA according to manufacturer's instructions (Trevigen, Gaithersburg, MD).

\section{Conformational changes of VLA-4 and LFA-1 integrins}

To assess VLA-4 conformational status in monocytes, the VLA-4-specific ligand, l-leucyl-l-aspartyl-1-valyl-l-prolyl-1alanyl-l-alanyl-l-lysine (LDV) (Tocris), was used. The activated conformation of VLA-4 was measured by flow cytometry (FACS) using HUTS21 Ab as described [8]. To assess LFA- 1 conformational status, monocytes $\left(0.5 \times 10^{6}\right.$ cells/ml in RPMI medium/1 \% FBS) were first treated with/out inhibitors and then stimulated with PMA (100 ng/ml) for $1 \mathrm{~h}$. To prevent RhoA or Rac1 GTPase activity, cells were pretreated with specific inhibitors, $1 \mu \mathrm{g} /$ ml CT04 (Cytoskeleton Inc.) or $75 \mu \mathrm{M}$ NSC23766 (EMD Millipore, Billerica, MA), respectively. Monocytes were placed on ice and fixed with $4 \%$ formaldehyde. Activated LFA-1 conformation was detected by FACS with MEM$148 \mathrm{Ab}$. Data were acquired with a FACS BD Canto II flow cytometer (BD Biosciences, San Jose, CA) and analyzed with FlowJo software (Tree Star, Inc., Ashland, OR). Data were collected from at least 10,000 events for each experimental condition and repeated with monocytes from three different donors. Quantitation of integrin conformational activation was performed, where the mean fluorescence intensity (MFI) of activated non-treated cells was assigned a value of 100 and the value of 0 was assigned to the MFI of cell autofluorescence.

\section{RhoA and Rac1 guanosine triphosphatase (GTPase) activity assay}

RhoA and Rac1 GTPase activities were measured by GLISA RhoA and Rac1 activation assay kits (Cytoskeleton Inc., Denver, $\mathrm{CO}$ ) in cell lysates prepared from monocytes (with/out PARPi) after stimulation with MCP-1 (30 ng/ml) or PMA (100 ng/ml) for $1 \mathrm{~h}$. To inhibit RhoA or Rac1 GTPase activity, cells were pretreated with specific inhibitors, $1 \mu \mathrm{g} / \mathrm{ml} \mathrm{CT04} \mathrm{(Cytoskeleton} \mathrm{Inc.)} \mathrm{or}$ $75 \mu \mathrm{M}$ NSC23766 (EMD Millipore, Billerica, MA), respectively. Monocytes stimulated with CN04 (Cytoskeleton Inc.) served as a positive control for Rho activity.

\section{Quantification of F-actin and G-actin}

Monocytes were treated with PARPi or RhoA/Rac1 inhibitors and stimulated by MCP-1 as described above, washed with ice-cold PBS, and fixed with $4 \%$ formaldehyde. Filamentous actin (F-actin) and globular actin (Gactin) were stained by Acti-stain ${ }^{\mathrm{TM}} 488$ phalloidin (Cytoskeleton) and DNase 1-Alexa 591 (Life Technologies), respectively. Data were collected from at least 20,000 events for each sample by a BD FACS Influx and analyzed as described above. The F/G actin ratio was calculated by dividing the mean fluorescent intensity (MFI) of F-actin by the MFI of G-actin.

\section{Monocyte adhesion assay}

BMVEC monolayers were treated overnight with TNF $\alpha$ (20 ng/ml). Monocytes were treated overnight with/out PARPi and washed prior to calcein-AM labeling. Fluorescence of adherent monocytes was measured using a Synergy 2 plate reader (BioTek, Winooski, VT) as described [8]. Results are presented as the mean \pm SEM fold adhesion (number of adherent monocytes for each experimental condition divided by the basal adhesion of the untreated control), which was assigned a value of 1 (7600 relative fluorescent units).

\section{Transendothelial migration assay}

Monocytes were treated with PARPi, labeled as described above, and washed before addition to BMVEC. 
FluoroBlok (BD Bioscience, Bedford, MA) cell culture inserts, intended to block the transmission of fluorescent light between 490 and $700 \mathrm{~nm}$, were used to permit continuous detection of fluorescently labeled monocytes migrating across endothelial monolayers. BMVEC were seeded on collagen type I coated $3-\mu \mathrm{m}$ pore 24-well tissue culture FluoroBlok inserts at a density of $2.5 \times 10^{4}$ cells per insert. Confluent monolayers were then exposed to TNF $\alpha(20 \mathrm{ng} / \mathrm{ml})$ for $24 \mathrm{~h}$ to activate the BMVEC. After activation, BMVEC were rinsed with fresh medium and the medium was replaced. For migration assays, calceinAM-labeled monocytes were added to the upper chamber of the tissue culture insert system, while the chemoattractant, CCL2/MCP-1 (30 ng/ml), was added to the lower chamber to stimulate migration. Chemotaxis towards MCP-1 was allowed for $2 \mathrm{~h}$ as described [8]. The number of migrated monocytes was determined using Image software $(\mathrm{NIH})$ and presented as fold difference in migration from triplicate determinations, calculated from the number of migrated monocytes for each experimental condition divided by the number of migrated monocytes in the untreated, no chemoattractant control (assigned a value of 1 , equivalent to 37 migrated cells).

\section{Transendothelial electrical resistance (TEER)}

To determine the integrity of brain endothelial monolayers after engagement with monocytes, TEER was measured using the 1600R ECIS system (Applied Biophysics, Troy, NY). Using the free ions in the culture media, the instrument generates an AC current flow between the electrode and counter electrode located in specialized tissue culture arrays and measures the change in impedance. The ECIS system provides realtime complex impedance, providing readouts for impedance, resistance, and capacitance. In brief, BMVEC were plated on collagen-coated electrode arrays (96W20idf) and maintained until a monolayer formed with a TEER of 800-1500 $\Omega$. Monocytes were treated with/out PARPi overnight, rinsed prior to the addition of monocytes $\left(1 \times 10^{5}\right.$ cells/well $)$ to the BMVEC. Measurements were taken every $30 \mathrm{~min}$ at $4000 \mathrm{~Hz}$ as described [39, 43]. The results are presented as the percent change from baseline TEER from at least two independent experiments (expressed as average \pm SEM) consisting of four to six replicates each $(100 \%$ equals a resistance of $644 \Omega$ ).

\section{Statistical analysis}

The results are expressed as the mean \pm SEM of experiments conducted multiple times. Multiple group comparisons were performed by one-way analysis of variance with Dunnet's post hoc tests. Statistical analyses were performed utilizing Prism v5 software (GraphPad Software Inc., La Jolla, CA). Differences were considered significant at $P$ values $<0.05$.

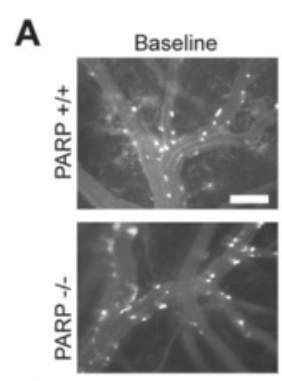

C

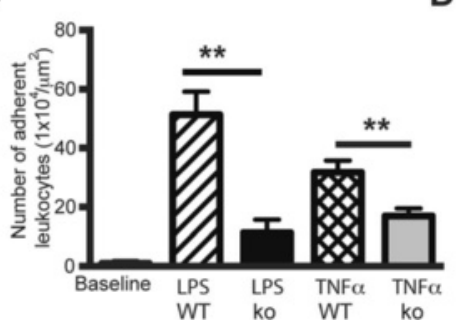

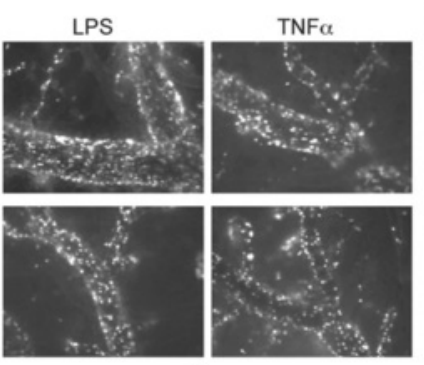

D

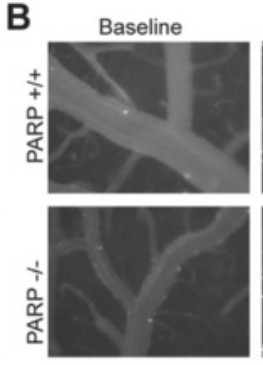

E
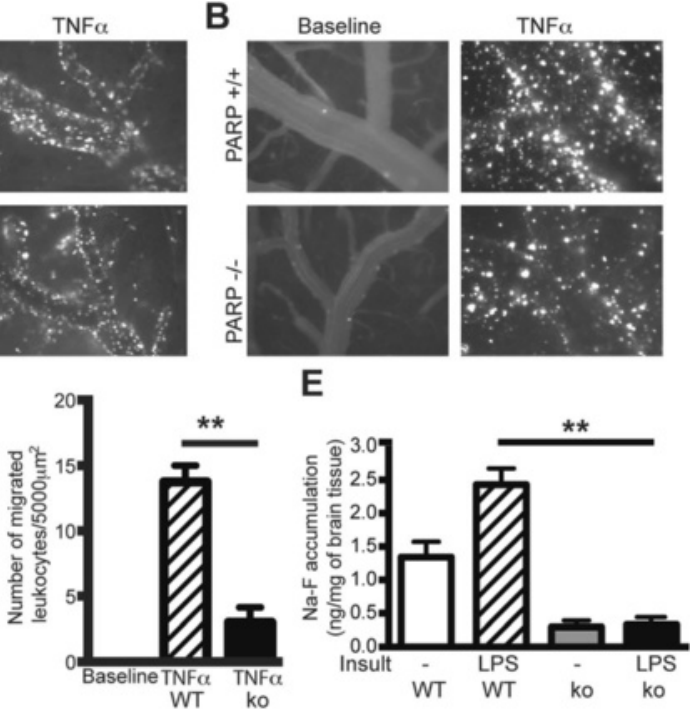

Fig. 1 PARP deficiency diminishes leukocyte adhesion to and migration across the BBB and reduces BBB permeability. Representative images from videos of leukocytes labeled with Rhodamine $6 \mathrm{G}$ for adhesion (a) and migration (b). Measurement of labeled leukocytes after their adhesion to (c) or migration (d) across the endothelium. e Measurement of BBB permeability in vivo by sodium-fluorescein accumulation in the brain in the presence or absence of LPS. Results are shown as mean adhesion or migration \pm SEM (four animals per treatment group). ${ }^{* *} P<0.01$ indicates significance vs. TNFa or LPS-treated animals. Scale bar $=100 \mu \mathrm{m}$ is shown in one micrograph, but it is valid for all micrographs shown 


\section{Results}

PARPko mice exhibit decreased leukocyte adhesion to and migration across the brain endothelium in vivo and attenuated enhanced BBB permeability in TNFa-induced encephalitis

Recently, we demonstrated that PARP inhibition in BMVEC lessened monocyte adhesion and migration across BMVEC monolayers and attenuated expression of adhesion molecules [35]. To further assess effects of PARP inhibition on leukocyte adhesion/migration, we used IVM in PARPko and WT mice, utilizing systemic LPS-induced inflammation [35] and TNF $\alpha$-mediated encephalitis (by IC injection). In WT mice, LPS and TNF $\alpha$ treatment resulted in 52- and 42-fold increases in leukocyte firm adhesion, respectively, whereas PARPko mice showed decreased level of leukocyte adhesion with both LPS (74 \%) and TNF $\alpha$ (25\%) (Fig. 1a, c).

IVM showed increased leukocyte migration across the brain endothelium after IC TNF $\alpha$ administration by 14.5-fold vs. baseline in WT mice, while PARPko mice displayed only a 2.6-fold increase vs. baseline (Fig. 1b, d). Because leukocyte engagement of endothelium and inflammation results in enhanced permeability of the $\mathrm{BBB}$, we determined the effect of PARP inhibition on barrier permeability in a LPS-induced systemic inflammation model. The amount of $\mathrm{Na}-\mathrm{F}$ in the whole brain was elevated 1.7-fold after LPS-treatment of WT mice compared to untreated WT mice (Fig. 1e) whereas in PARPko mice, Na-F accumulation was significantly decreased in untreated and LPS-treated PARPko animals as compared to WT mice (Fig. 1e). These results further indicate that suppression of PARP activity protects $\mathrm{BBB}$ integrity.

Inhibition of PARP decreases leukocyte adhesion to the brain endothelium in an adoptive transfer mouse model of LPS-induced systemic inflammation

Our previous work utilizing systemic PARPi administration [35] or PARPko animals (Fig. 1) did not allow us to determine whether the effects observed were due to diminution of inflammatory responses in endothelial cells or in leukocytes. Therefore, we evaluated the effect

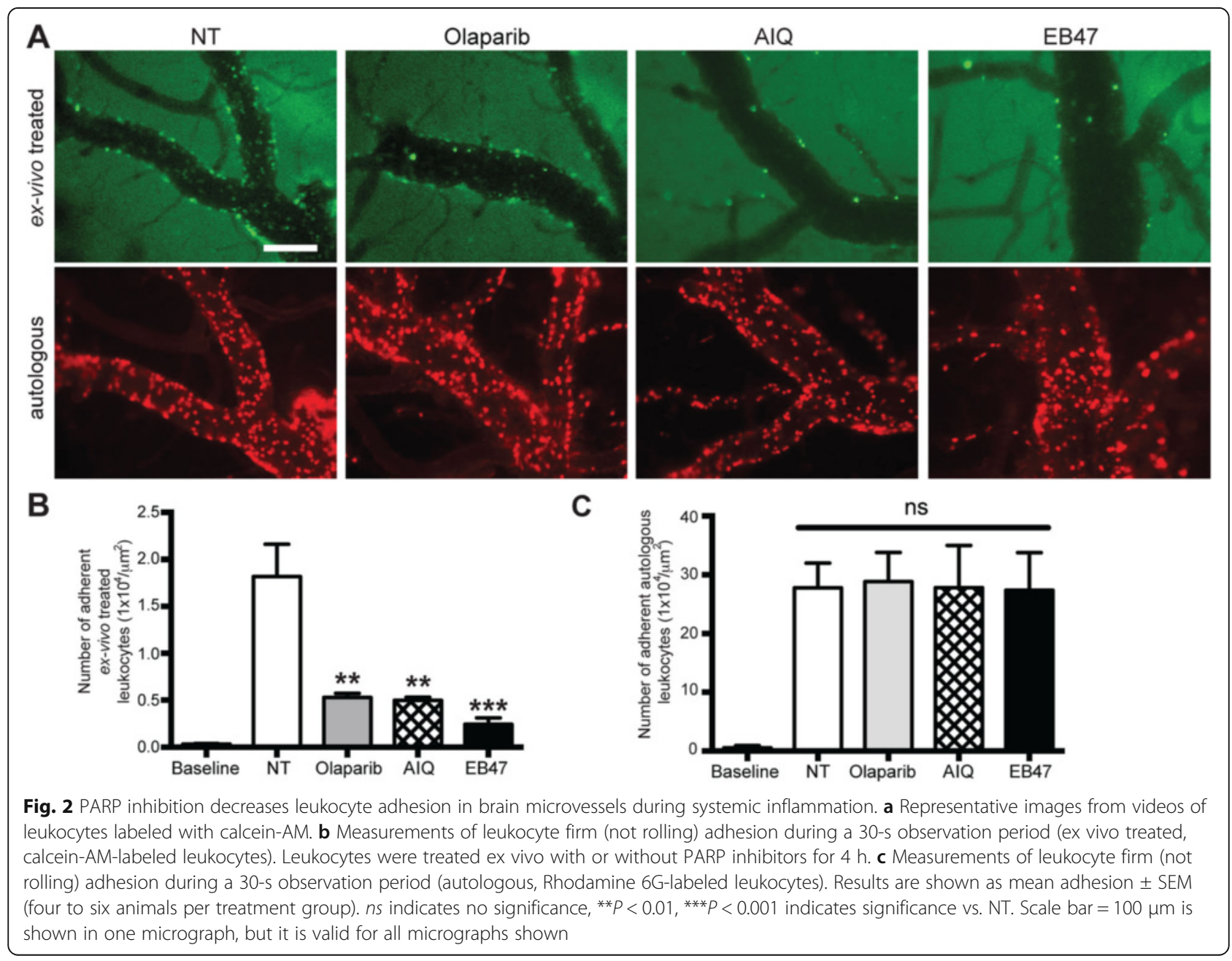


of selective PARP inhibition in leukocytes on their adhesion in vivo utilizing adoptive transfer. Leukocytes were isolated from WT mice, treated ex vivo with/out PARPi, labeled with calcein-AM, washed, injected into LPStreated WT mice, and monitored by IVM. Olaparib and EB47 ex vivo treatment reduced PARP activity to levels close to PARPko leukocytes, whereas AIQ reduced PARP activity by $53 \%$ (Additional file 1: Figure S2A). LPS treatment increased leukocyte attachment (adhesion) $>40$-fold to the brain endothelium, while leukocytes ex vivo treated with PARPi displayed significantly decreased adhesion compared to untreated leukocytes by $71-87 \%$ (Fig. 2a, b). Mice injected with LPS showed $~ 30$-fold increase in firm adhesion of autologous (non-treated) leukocytes to the cortical endothelium (Fig. 2c). These results demonstrate that PARPi have potent in vivo anti-inflammatory properties in leukocytes.

\section{PARP inactivation in leukocytes attenuates adhesion to} the brain endothelium

Since administration of PARPi significantly reduced leukocyte adhesion to cortical vessels, we hypothesized that PARP inhibition would affect the ability of leukocytes to adhere to cortical vessels. We performed adoptive transfer where ex vivo-labeled leukocytes, isolated from PARPko animals, were i.v.-injected into recipient WT animals and vice versa, utilizing a LPS-induced systemic encephalitis model. PARPko leukocytes transferred to WT mice exhibited $36 \%$ decreased adhesion compared to WT PARP-expressing leukocytes injected to WT mice (Fig. 3a, b). When PARPko leukocytes were

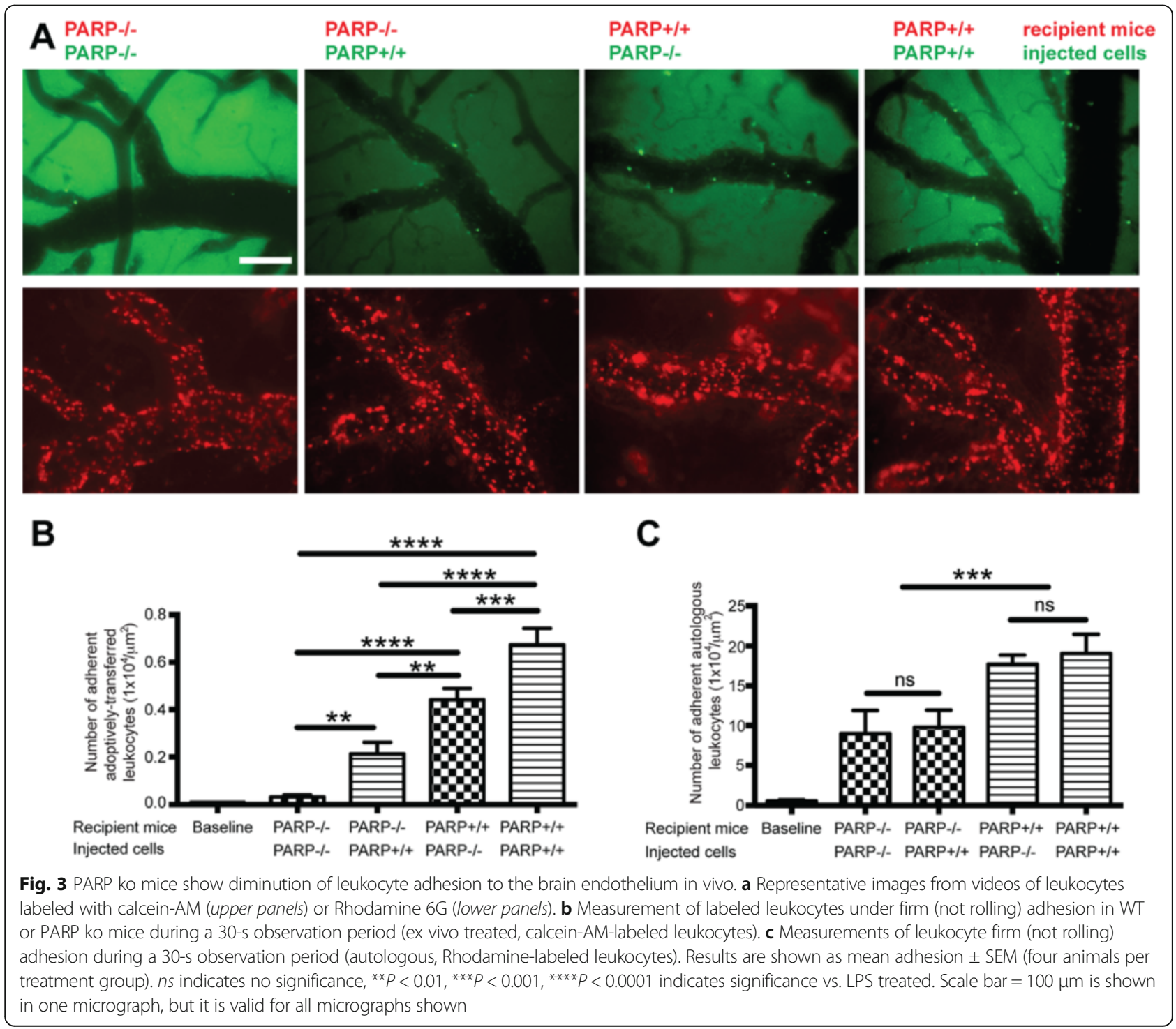




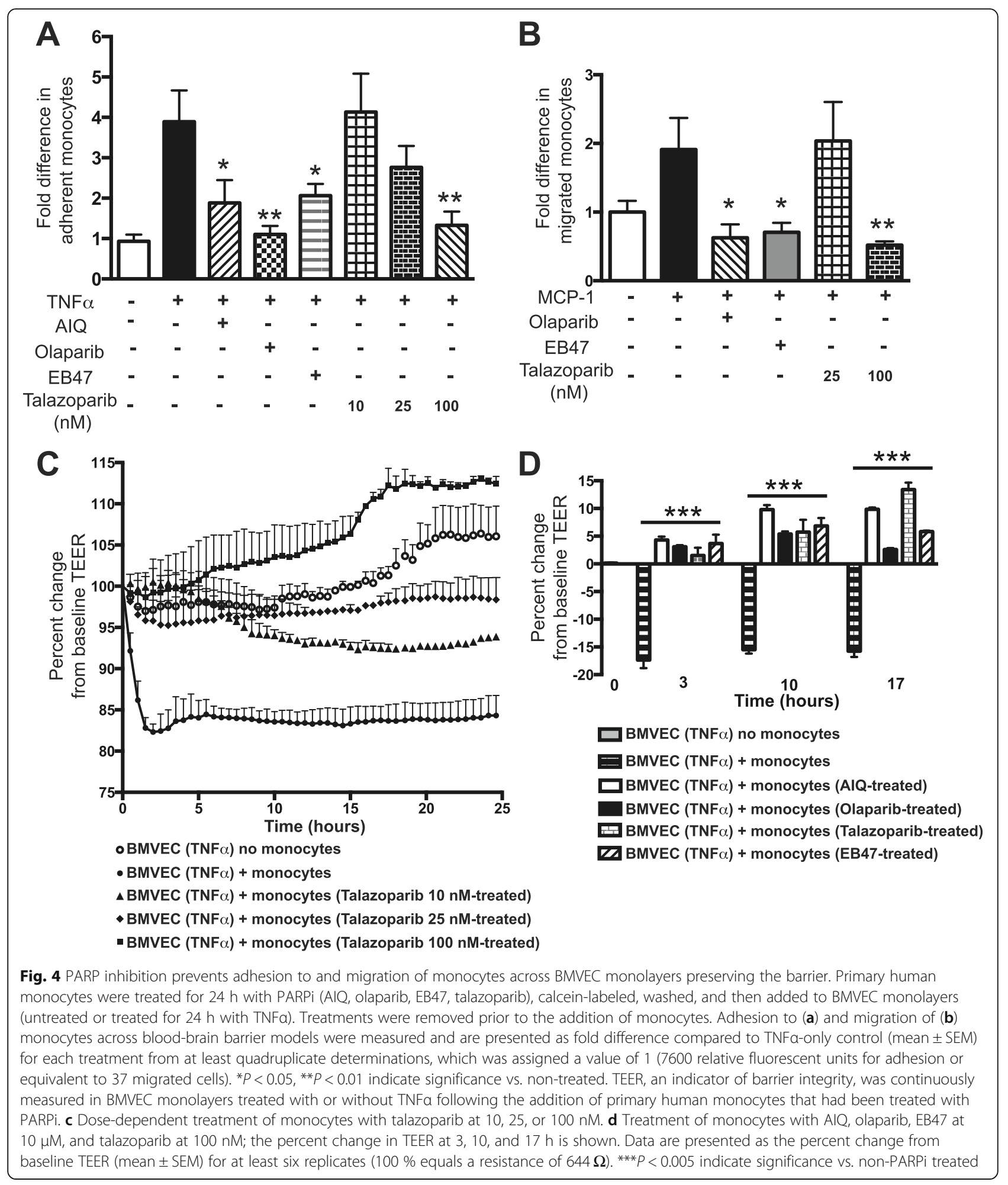

transferred to PARPko mice, leukocytes showed even further decrease in adhesion. Whereas the cells from the PARPko mice were able to adhere much better in the WT mice compared to the PARPko mice mimicking results in Fig. 1c, and leukocyte adhesion in the PARPko animals was reduced too. Adhesion of autologous leukocytes did not change, regardless of the type of transferred leukocytes (Fig. 3c). These results support our idea that inhibition of PARP in leukocytes affects their capability to adhere to endothelium. 


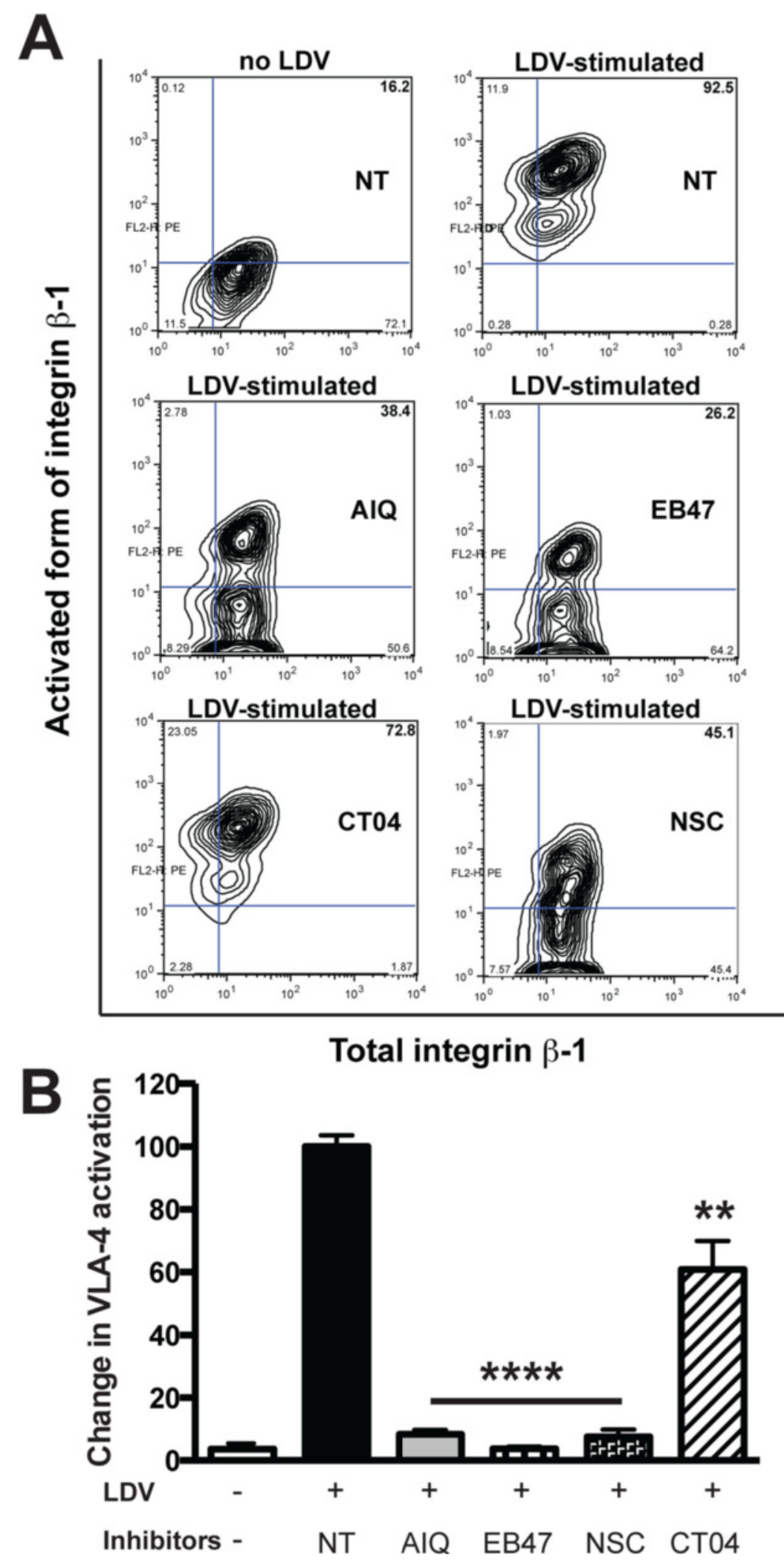

Fig. 5 (See legend on next page.) 
(See figure on previous page.)

Fig. 5 PARP inhibition prevents conformational activation of VLA-4 (integrin $\beta 1$ ) in monocytes. Monocytes were treated with PARPi (AIQ or EB47) or RhoA or Rac1 GTPase inhibitor (CT04 or NSC23766). The expression of total VLA-4 (CD29) was unaffected by treatment with PARP inhibitors. a The active form of VLA-4 was detected using HUTS21, a conformation-specific antibody. Integrin conformation in monocytes changes from a closed (inactive) to an open (active) form after LDV stimulation. Total amount of integrin $\beta 1$ (CD29) is shown on the $x$-axis. b Quantitation of integrin $\beta 1$ conformational activation, expressed as the percent of monocytes containing the activated integrin conformation (as shown in the right upper quadrant of each graph in panel a). Results are presented as the mean \pm SEM (**P $<0.01,{ }^{* * *} P<0.005$ vs. untreated control) from three independent experiments

\section{PARP suppression in monocytes decreases adhesion to BMVEC monolayers and transendothelial migration}

We determined whether our in vivo observations could be confirmed in an in vitro BBB system utilizing primary human monocytes and human BMVEC. Monocytes were treated with PARPi, washed and added to TNF $\alpha$ stimulated BMVEC. Monocyte adhesion to BMVEC was increased nearly fourfold following TNF $\alpha$ treatment and was significantly inhibited by the PARPi, AIQ, Olaparib, and EB47 (by 55, 91, and $47 \%$, respectively) (Fig. 4a). In dose-response experiments, talazoparib reached a significant (86 \%) decrease in adhesion at $100 \mathrm{nM}$. We next determined if PARP inhibition in monocytes would affect their transendothelial migration through BMVEC monolayers. Monocytes were treated with PARPi; after treatments were removed, MCP-1 was added to the lower chamber of $\mathrm{BBB}$ constructs, which resulted in a twofold increase in monocyte migration towards the chemoattractant. Monocyte migration was completely abolished by PARPi, olaparib, EB47, and talazoparib (Fig. 4b). These in vitro protective effects observed with PARPi on monocyte/endothelial interactions in adhesion/migration assays are consistent with our in vivo results.

\section{PARP inhibition prevents monocyte-mediated disruption of the BBB}

To understand if PARP inhibition in monocytes could diminish their activation, lessen BMVEC engagement, and preserve barrier integrity in brain endothelial cells in vitro, we used TEER to assess barrier tightness in BMVEC monolayers. BMVEC demonstrated the expected steady state barrier formation, but monocyte addition resulted in a rapid diminution (15-20 \%) in resistance (mimicking BBB injury in vivo) that was maintained for the experiment's duration. Dose-response monocyte exposure to talazoparib, significantly attenuated monocyte engagementinduced drop in resistance, enhancing TEER by 7-10, 10-13, and 17-27 \% (Fig. 4c). AIQ, olaparib, and EB47 also prevented barrier injury, as demonstrated by $3-10 \%$ increases in TEER (Fig. 4d). Taken together, these results demonstrate that PARP inhibition in monocytes decreases their engagement with endothelium and protects barrier integrity in vitro.
Inhibition of PARP prevents conformational activation of VLA-4 and LFA-1 integrins in monocytes

The adhesion of monocytes to activated endothelial cells is governed by conformational changes in the integrins, including VLA-4 and LFA-1 [8]. We evaluated expression of active forms of VLA-4 following stimulation with LDV (a peptide mimicking activation during exposure to the adhesion molecule, VCAM-1, and fibronectin), which resulted in a change in integrin $\beta 1$ conformation from inactive (closed/non-adherent) to active (open/adherent) form. PARPi treatment of monocytes drastically decreased LDV-induced expression of active integrin $\beta 1$, as did treatment with Rac1 inhibitor, NSC23766 (>90\%) (Fig. 5). The RhoA inhibitor, CT04, decreased this to a lesser degree (40\%).

Activation of primary human monocytes by PMA resulted in a significant twofold upregulation of the active form of LFA-1, while exposure to olaparib, EB47, or talazoparib resulted in a significant decline of activated LFA-1 compared to untreated monocytes (Fig. 6). Rac1 inhibitor treatment also significantly decreased LFA-1 activation $(P<0.05)$. However, RhoA inhibitor treatment reduced LFA-1 activation with lesser extent $(P<0.5)$.

PARP inactivation in monocytes affects actin cytoskeleton We next measured PARP inhibition effects on actin cytoskeleton rearrangements in monocytes. MCP-1stimulation of monocytes resulted in a 5.6-fold increase in $\mathrm{F} / \mathrm{G}$ actin ratio (Fig. 7a). PARPi treatment of monocytes resulted in a $28 \%$ decrease in $\mathrm{F} / \mathrm{G}$ action ratio. Specific RhoA and Rac1 inhibitors also decreased $F / G$ actin ratio, indicating that PARP inhibition affects the actin cytoskeleton via RhoA and Rac1 inhibition. We next evaluated the activation of the small GTPases, RhoA, and Rac1, in monocytes, in view of their role in the actin cytoskeleton rearrangements during their adhesion and migration. MCP-1-stimulated monocytes showed increased RhoA by 1.6 (Fig. 7b). MCP-1- or PMA-stimulated monocytes exhibited amplified Rac1 activation by 4and 5.9-fold, respectively (Fig. 7c). PARPi, olaparib, and talazoparib significantly reduced activation of both Rho GTPases. 


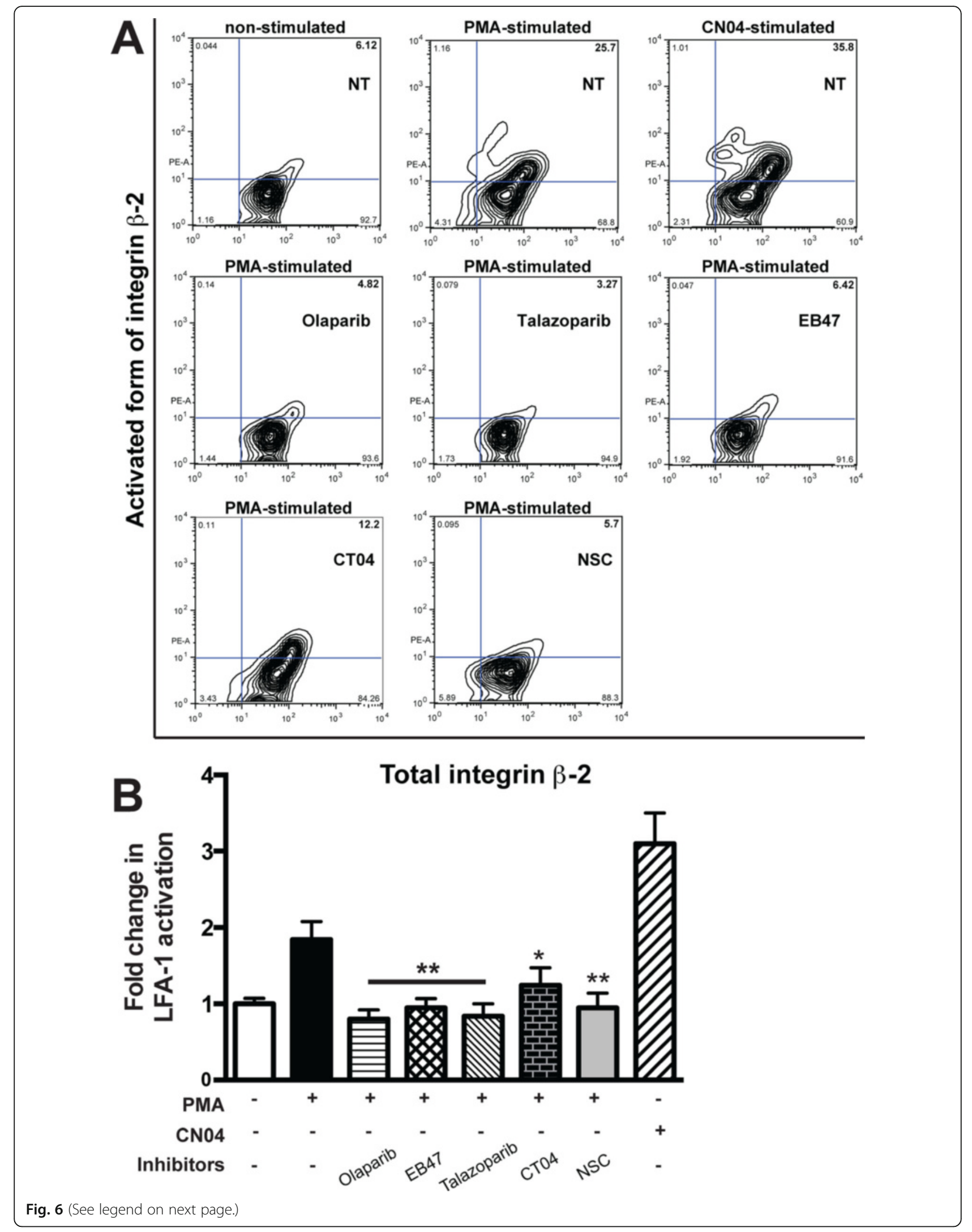


(See figure on previous page.)

Fig. 6 PARP suppression diminishes conformational activation of LFA-1 (integrin $\beta 2$ ) in monocytes. Monocytes were treated with PARPi (olaparib, EB37, or talazoparib) or RhoA and Rac1 GTPase inhibitor (CT04 or NSC23766). Expression of total LFA-1 (CD18) was not affected by treatment with PARPi. The activated form of LFA-1 was detected using MEM-148, a conformation-specific antibody. PMA-stimulation of monocytes changes integrin conformation from a closed (inactive) to an open (active) form (a). The total amount of integrin $\beta 2$ (CD18) is shown on the $x$-axis. $\mathbf{b}$ Quantitation of integrin $\beta 2$ conformational activation, expressed as the percent of monocytes containing the activated integrin conformation (as shown in the right upper quadrant of each graph in panel a). Results are presented as the mean \pm SEM $\left({ }^{*} P<0.5,{ }^{* *} P<0.05\right.$ vs. untreated control) from three independent experiments

\section{Discussion}

Leukocyte trafficking from blood into tissues is a vital response to injury and involves multiple steps mediated by adhesion molecules and chemoattractants. Inflammation and its associated infiltration of various leukocyte types, as well as leukocyte-endothelium interaction, play a significant role in numerous CNS diseases; therefore, the molecular mechanisms controlling leukocyte migration are potential therapeutic targets. Inflammatory and immune cells play complex roles after ischemic stroke, MS, encephalitis, and meningitis [10, 19, 45-48]. Actin cytoskeleton machinery, integrins, and their conformational changes are key elements in the ability of leukocyte subsets to roll on endothelial cells, arrest and firmly adhere, and transmigrate across the BBB, leading to its dysfunction and neuroinflammation resulting in neuronal dysfunction. Lessening leukocyte adhesion and migration might serve as an ultimate tool in weakening of inflammatory events in the brain.

PARP suppression has been shown to diminish edema, preserve the tight junction protein, occludin, and decrease expression of the adhesion molecule, ICAM-1, in animal models of stroke, traumatic brain injury, meningitis, and MS [45, 49-51], reducing leukocyte infiltration and brain inflammation $[49,52]$. Application of PARPi has been previously shown to reduce leukocyte adhesion $[49,52]$. However, these studies did not address whether the effects of PARP inactivation apply to immune cells, to endothelium or to both. Recently, we showed that PARP inhibition caused a significant reduction in leukocyte adhesion/migration in a novel in vivo model of localized neuroinflammation as well as in systemic inflammation, thereby preserving BBB integrity [35]. Additionally, inhibition of PARP in BMVEC resulted in attenuation of expression of adhesion molecules, such VCAM-1 and ICAM-1, and diminished secretion of proinflammatory cytokines [35].

In the present study, we used IVM to demonstrate that PARP inactivation in leukocytes drastically attenuated leukocyte adhesion/migration to and across cortical vessels in two in vivo models, LPS-induced systemic inflammation, and TNF $\alpha$-induced localized encephalitis (Fig. 2), whereas autologous leukocytes displayed regular adhesion. PARPko animals exhibited attenuated inflammationcaused permeability vs. WT mice (Fig. 1c). PARP absence resulted in a decreased number of adherent leukocytes when injected into WT mice or an enhanced number of adherent leukocytes when WT leukocytes were introduced into PARPko mice (Fig. 3). Interestingly, the cells from the PARPko mice were able to adhere much better in the WT mice vs. the PARPko mirroring results in Fig. 1c, whereas leukocyte adhesion in the PARPko animals was reduced too, not only the leukocyte adhesion machinery was affected in these animals but also the BBB was less impaired [35]. Our in vivo results were reproduced in vitro where PARPi-treated monocytes showed substantially reduced adhesion to and migration across BMVEC monolayers (Fig. 4). The present study determined that inactivation of PARP in primary human monocytes tempered barrier dysfunction (denoted by a TEER reduction) (Fig. 4), with diminished monocyte engagement of the brain endothelium (adhesion/migration).

We have explored the idea that PARP inactivation results in conformational changes of active integrin and/or total integrin expression. Inactivation of PARP and Rac1 inhibition attenuated active integrin $\beta 1$ expression (Fig. 5), while expression of total VLA-4 was unchanged, demonstrating the importance of VLA-4 conformation. Conformational activation of integrin apparently exposes the VCAM-1 binding site [53], facilitating both tethering and rolling [54]. It has been shown that memory T cells, constitutively expressing activation/ligand-induced epitopes on $\beta 1$ integrins, exhibit significantly higher rates of attachment and accumulation on VCAM-1 expressing cells compared to other T cell subsets without active epitope expression $[9,55]$. Previously, we $[8,9]$ and others [56] have shown a link between conformational VLA-4 activation and Rac1 pathways (inhibition of GSK3 $\beta$ or Rac-1, or CB2 activation), leading to lessened expression of the active VLA-4 form resulted in attenuated monocyte adhesion/migration in BMVEC $[8,9]$.

LFA-1 inside-out activation was stimulated by PMA in primary monocytes, and Rac1 inhibition or PARP inactivation decreased LFA-1 conformational change, whereas RhoA inhibition had no effect (Fig. 6). It has been shown that Rac1 plays an important role in LFA-1 activation in $\mathrm{T}$ cells [57]. Mutations of the LFA-1 $\beta 2$-subunit prevented LFA-1 surface expression [58], resulting in type I leukocyte adhesion deficiency, which is induced by small Rho GTPase signaling [59]. 


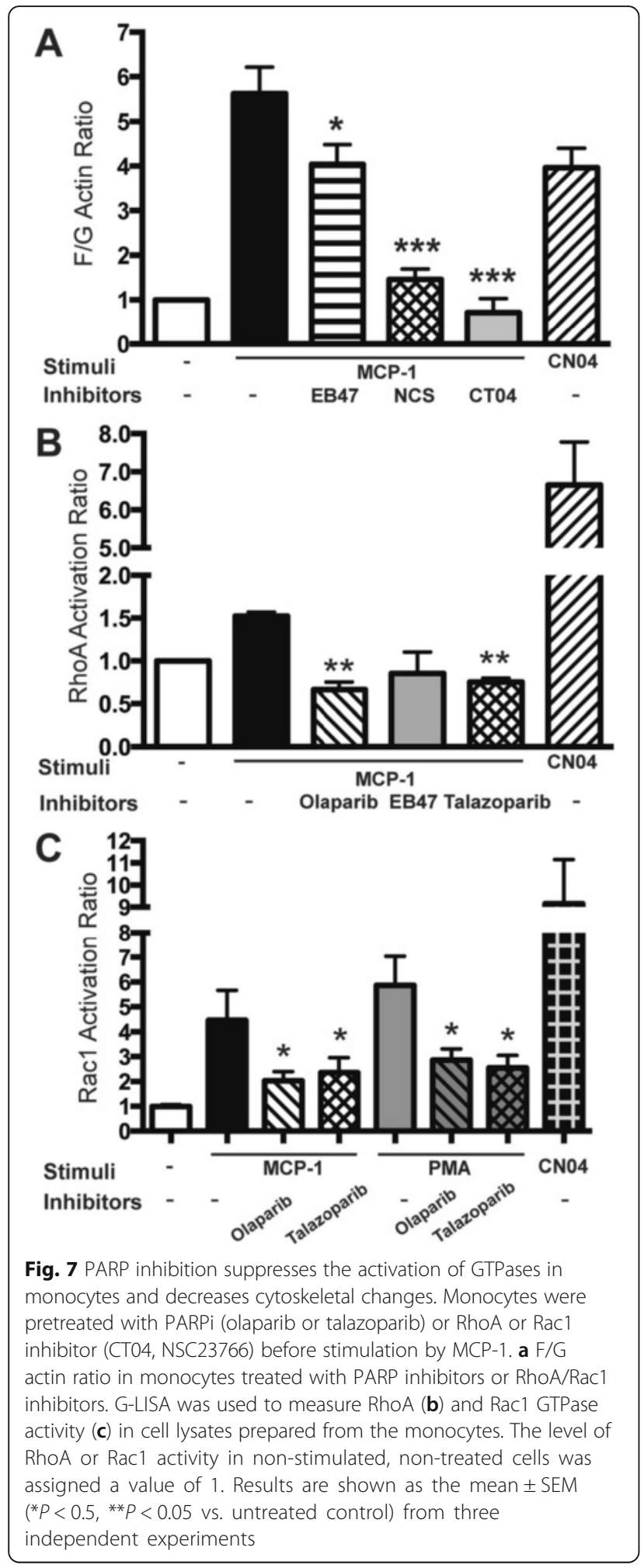

Small GTPases (Rho, Rac, and Cdc42) play a vital role in transendothelial leukocyte migration, oxidative stress, and inflammation by linking surface receptors and the actin cytoskeleton $[60,61]$. Rac1 and Cdc42 in leukocytes control lamellipodia formation, cell polarity, and direct migration, while RhoA controls leukocyte tail retraction during transmigration [62]. Given outcomes of functional assays, we measured RhoA and Rac1 activation after MCP-1 or PMA stimulation of monocytes and showed a decrease in active GTPases after PARP inactivation, resulting in diminution of actin cytoskeletal rearrangements (Fig. 7), which play a central role in cell migration [8, 63-65]. F-actin-rich lamellipodia formation caused by stimulating actin filament disassembly near the pointed ends supplies actin monomers for polymerization [66]. MCP-1 stimulation resulted in amplified actin rearrangements in monocytes (high $\mathrm{F} / \mathrm{G}$ actin ratio), whereas PARP inactivation substantially diminished F/G actin ratio (Fig. 7). RhoA and Rac1 inhibitors exhibited analogous results, further signifying their involvement in this process.

There is a good amount of literature suggesting that PARP serves as a key component in nuclear factor kappa $\mathrm{B}$, NFkB, activation, and consequently to transcription and expression of many inflammatory genes [35, 39, 6769]. In brain-resident microglia, TNF $\alpha$ activates PARP in a DNA damage-independent manner, involving phosphatidyl choline-specific phospholipase and the MAPK kinase, ERK [70].

Rho GTPases are known to participate in MAPK kinase signaling activation [71]. In hepatocytes, MAPK kinase downregulation significantly abrogated the Rho pathway and cytoskeleton reorganization [72]. It is widely accepted that there is a relationship between PARP and MAPK, suggesting that they might stimulate each other in a feedback cycle [73]. Both MAPK kinases and Rho GTPases are involved in cytoskeleton rearrangements and cell migration $[9,71,74]$. Deeper understanding of these signal transduction interrelations may contribute to the development of more fine-tuned antiinflammatory therapeutics.

Oxidative stress plays an important role in many neuroinflammatory conditions $[75,76]$. Prolonged oxidative stress might lead to alterations in actin cytoskeleton in leukocytes [77]. In recent years, numerous PARP functions have been discovered, involving the regulation of oxidative stress and inflammation via complex interlinked signaling networks [73, 78]. Free radicals are potent activators of PARP; consequently, augmented PARP activity is usually associated with oxidative/nitrative stress [79]. Although the latest reports have implicated a bidirectional relationship, PARP inhibition itself is capable of reducing ROS formation in various pathologies $[51,79,80]$. PARP activation affects the energy balance of cells by $\mathrm{NAD}^{+}$ depletion, thereby causing compromised glycolysis and ATP depletion [78]. Functional behavior of immune cells (macrophages) is intensely coordinated by 
actin cytoskeleton rearrangements, a process in which ATP and $\mathrm{NAD}^{+}$also play an indispensable role [81]. In this report, we did not study PARP activationstimulated energy depletion and its effects on leukocyte function. However, understanding the role of PARP-stimulated $\mathrm{NAD}^{+}$depletion and leukocyte actin cytoskeleton machinery during inflammation is required in the future. Two PARPi that were used in this study, talazoparib and olaparib, are now in clinical trials for treatment of several cancers (phase II and III) [10], promising speedy translation in evolving therapeutic route in various inflammatory diseases [82]. While the anti-inflammatory effects of PARP inhibition are gaining momentum [83], exact mechanisms are still largely unknown (especially in the CNS).

The concept that BBB damage is tied to leukocyte migration into the CNS is well accepted [28]. Therefore, attenuated adhesion of inflammatory cells to the brain endothelium via inhibition of PARP may prove valuable in stroke, MS or infection-associated encephalitis, and other inflammatory disorders. Particularly for the field of stroke, the inflammatory response has Janus-faced effects. Inflammation is known to have deleterious effects [32, 84-86], but is also known to initiate regenerative processes, leading to the perspective that inflammation is a necessary part of the physiological reaction towards tissue repair and plasticity [32-34]. In this perspective, effects resulting in BBB opening after an acute stimulus (during ische$\mathrm{mia} /$ reperfusion) and PARP inhibition might be very valuable, but long-term effects of PARP inhibition need to be investigated further before such an intervention could be considered clearly beneficial. Here, we have described previously unrecognized effects of PARP inhibition on diminution of VLA-4 and LFA-1 activation, mitigation of actin cytoskeleton rearrangements via small GTPases, and attenuation of leukocyte adhesion and migration across the BBB. Attenuation of leukocyte invasion is associated with minimized brain damage and might serve to translate these experimental findings into clinical therapies.

\section{Conclusions}

Neuroinflammation is accompanied by leukocyte infiltration into the brain resulting in subsequent $\mathrm{BBB}$ dysfunction and significantly contributing to morbidity in ischemic stroke, multiple sclerosis, encephalitis, and traumatic brain injury. Pathways that reduce the inflammatory potential of leukocytes would prevent such damage. Our results suggest PARP inhibition in leukocytes as a novel approach to BBB protection in the setting of endothelial dysfunction caused by inflammation-induced leukocyte engagement.

\section{Additional file}

Additional file 1: Figure S1. PARPi decrease PARP activity in a dosedependent manner. PARP activity of primary monocytes treated with different concentrations of PARPi AIQ (A) and olaparib (B). Results are presented as the mean \pm SEM $\left({ }^{*} P<0.05,{ }^{* *} P<0.01\right.$ vs. untreated control) from two independent experiments for at least three replicates. Figure S2. PARP activity significantly diminished in ex vivo treated mouse leukocytes. (A) PARP activity was measured in freshly isolated and ex vivo PARPi-treated mouse leukocytes. Data are presented as mean \pm SEM for at least three replicates from three/four donor mice. ${ }^{* * *} P<0.005$ indicate significance vs. non-treated. (B) Flow cytometry data presenting leukocyte profile of isolated leukocytes with or without PARPi treatment.

\section{Abbreviations}

AIQ-5: Aminoisoquinolinone; BBB: Blood-brain barrier; BMVEC: Brain microvascular endothelial cells; EB47: 5'-Deoxy-5'-[4-[2-[(2, 3-dihydro-1-oxo1H-isoindol-4-yl) amino]-2-oxoethyl]-1-piperazinyl]-5'-oxoadenosine dihydrochloride; FACS: Fluorescence-activated cell sorting; IC: Intracerebral; ICAM-1: Intercellular adhesion molecule 1; IVM: Intravital videomicroscopy; LPS: Lipopolysaccharide; MCP-1: Monocyte chemotactic protein-1; MFI: Mean fluorescence intensity; MS: Multiple sclerosis; MS: Multiple sclerosis; NaF: Sodium fluorescein; NFkB: Nuclear factor kappa B; PARP: Poly(ADP-ribose) polymerase; PARPi: Poly(ADP-ribose) polymerase inhibitors; TBI: Traumatic brain injury; TEER: Transendothelial electrical resistance; VCAM-1: Vascular cell adhesion molecule 1

\section{Acknowledgements}

Not applicable.

\section{Funding}

The work performed in the authors' laboratories is supported by grants from MH65151 (YP), AA015913 (YP), DA013429 (YP), NS087385 (SR), and ZIAAA000375 (PP). The fund agencies had no role in study design, data collection and analysis, decision to publish, or preparation of the manuscript.

Availability of data and materials

Data supporting the conclusions of this article are presented in the manuscript.

\section{Authors' contributions}

SR, VZR, NR, HD, SG, and YP have made substantial contributions to conception and design, acquisition of data, and analysis and interpretation of data and have given final approval of the version to be published and agree to be accountable for all aspects of the work in ensuring that questions related to the accuracy or integrity of any part of the work are appropriately investigated and resolved. SR, NR, PP, and YP have been involved in drafting the manuscript and revising it critically for important intellectual content.

\section{Competing interests}

The authors declare that they have no competing interests.

Consent for publication

Not applicable.

\section{Ethics approval}

This study did not utilize any human participants, human data, or human tissue. Animal experiments were approved by the Temple University Institutional Animal Care and Use Committee in accordance with guidelines based on the National Institutes of Health $(\mathrm{NIH})$ guide for care and use of laboratory animals.

\section{Author details}

'Department of Pathology and Laboratory Medicine, Temple University, Philadelphia, PA 19140, USA. ${ }^{2}$ Center for Substance Abuse Research, Lewis Katz School of Medicine, Temple University, Philadelphia, PA 19140, USA. ${ }^{3}$ Laboratory of Cardiovascular Physiology and Tissue Injury, National Institutes of Health/NIAAA, Bethesda, MD 20852, USA. 
Received: 29 July 2016 Accepted: 21 September 2016 Published online: 27 September 2016

\section{References}

1. Allport JR, Ding H, Collins T, Gerritsen ME, Luscinskas FW. Endothelialdependent mechanisms regulate leukocyte transmigration: a process involving the proteasome and disruption of the vascular endothelialcadherin complex at endothelial cell-to-cell junctions. J Exp Med. 1997;186: 517-27.

2. Alon R, Feigelson SW, Manevich E, Rose DM, Schmitz J, Overby DR, Winter E, Grabovsky V, Shinder V, Matthews BD, et al. Alpha4beta1-dependent adhesion strengthening under mechanical strain is regulated by paxillin association with the alpha4-cytoplasmic domain. J Cell Biol. 2005;171:1073-84.

3. Chigaev A, Waller A, Amit O, Halip L, Bologa CG, Sklar LA. Real-time analysis of conformation-sensitive antibody binding provides new insights into integrin conformational regulation. J Biol Chem. 2009;284:14337-46.

4. Hogg N, Patzak I, Willenbrock F. The insider's guide to leukocyte integrin signalling and function. Nat Rev Immunol. 2011;11:416-26.

5. Evans R, Lellouch AC, Svensson L, McDowall A, Hogg N. The integrin LFA-1 signals through ZAP-70 to regulate expression of high-affinity LFA-1 on T lymphocytes. Blood. 2011;117:3331-42.

6. Chigaev A, Sklar LA. Aspects of VLA-4 and LFA-1 regulation that may contribute to rolling and firm adhesion. Front Immunol. 2012;3:242.

7. Cernuda-Morollon E, Ridley AJ. Rho GTPases and leukocyte adhesion receptor expression and function in endothelial cells. Circ Res. 2006:98:757-67.

8. Rom S, Fan S, Reichenbach N, Dykstra H, Ramirez SH, Persidsky Y. Glycogen synthase kinase 3beta inhibition prevents monocyte migration across brain endothelial cells via Rac1-GTPase suppression and down-regulation of active integrin conformation. Am J Pathol. 2012;181:1414-25.

9. Rom S, Zuluaga-Ramirez V, Dykstra H, Reichenbach NL, Pacher P, Persidsky Y. Selective activation of cannabinoid receptor 2 in leukocytes suppresses their engagement of the brain endothelium and protects the blood-brain barrier. Am J Pathol. 2013;183:1548-58.

10. Cavone L, Aldinucci A, Ballerini C, Biagioli T, Moroni F, Chiarugi A. PARP-1 inhibition prevents CNS migration of dendritic cells during EAE, suppressing the encephalitogenic response and relapse severity. Mult Scler. 2011;17:794-807.

11. Martinez-Zamudio Rl, Ha HC. PARP1 enhances inflammatory cytokine expression by alteration of promoter chromatin structure in microglia. Brain Behav. 2014;4:552-65.

12. Martire S, Mosca L, d'Erme M. PARP-1 involvement in neurodegeneration: a focus on Alzheimer's and Parkinson's diseases. Mech Ageing Dev. 2015;146148:53-64.

13. Ahmad SF, Zoheir KM, Ansari MA, Korashy HM, Bakheet SA, Ashour AE, AlShabanah OA, Al-harbi MM, Attia SM. The role of poly (ADP-ribose) polymerase-1 inhibitor in carrageenan-induced lung inflammation in mice. Mol Immunol. 2015;63:394-405

14. Canan S, Maegley K, Curtin N. Strategies employed for the development of PARP inhibitors. Methods Mol Biol. 2011;780:463-89.

15. Wahlberg E, Karlberg T, Kouznetsova E, Markova N, Macchiarulo A, Thorsell AG, Pol E, Frostell A, Ekblad T, Oncu D, et al. Family-wide chemical profiling and structural analysis of PARP and tankyrase inhibitors. Nat Biotechnol. 2012:30:283-8.

16. Qiu W, Lam R, Voytyuk O, Romanov V, Gordon R, Gebremeskel S, Vodsedalek J, Thompson C, Beletskaya I, Battaile KP, et al. Insights into the binding of PARP inhibitors to the catalytic domain of human tankyrase-2. Acta Crystallogr D Biol Crystallogr. 2014;70:2740-53.

17. d'Avila JC, Lam TI, Bingham D, Shi J, Won SJ, Kauppinen TM, Massa S, Liu J, Swanson RA. Microglial activation induced by brain trauma is suppressed by post-injury treatment with a PARP inhibitor. J Neuroinflammation. 2012;9:31.

18. Kauppinen TM, Suh SW, Genain CP, Swanson RA. Poly (ADP-ribose) polymerase- 1 activation in a primate model of multiple sclerosis. J Neurosci Res. 2005;81:190-8.

19. Koedel U, Winkler F, Angele B, Fontana A, Pfister HW. Meningitis-associated central nervous system complications are mediated by the activation of poly (ADP-ribose) polymerase. J Cereb Blood Flow Metab. 2002;22:39-49.

20. Ahmad SF, Zoheir KM, Bakheet SA, Ashour AE, Attia SM. Poly (ADP-ribose) polymerase- 1 inhibitor modulates $T$ regulatory and IL-17 cells in the prevention of adjuvant induced arthritis in mice model. Cytokine. 2014;68:76-85.
21. Horvath B, Magid L, Mukhopadhyay P, Batkai S, Rajesh M, Park O, Tanchian G, Gao RY, Goodfellow CE, Glass M, et al. A new cannabinoid CB2 receptor agonist HU-910 attenuates oxidative stress, inflammation and cell death associated with hepatic ischaemia/reperfusion injury. Br J Pharmacol. 2012; 165:2462-78.

22. Pasqualetti G, Brooks DJ, Edison P. The role of neuroinflammation in dementias. Curr Neurol Neurosci Rep. 2015;15:17.

23. Lecuyer MA, Kebir $\mathrm{H}$, Prat A. Glial influences on BBB functions and molecular players in immune cell trafficking. Biochim Biophys Acta. 1862;2015:472-82.

24. Persidsky Y, Ramirez SH, Haorah J, Kanmogne GD. Blood-brain barrier: structural components and function under physiologic and pathologic conditions. J Neuroimmune Pharmacol. 2006;1:223-36.

25. Gorina R, Lyck R, Vestweber D, Engelhardt B. beta2 integrin-mediated crawling on endothelial ICAM-1 and ICAM-2 is a prerequisite for transcellular neutrophil diapedesis across the inflamed blood-brain barrier. J Immunol. 2014;192:324-37.

26. Gelderblom M, Leypoldt F, Steinbach K, Behrens D, Choe CU, Siler DA Arumugam TV, Orthey E, Gerloff C, Tolosa E, Magnus T. Temporal and spatial dynamics of cerebral immune cell accumulation in stroke. Stroke. 2009;40:1849-57.

27. Stevens SL, Bao J, Hollis J, Lessov NS, Clark WM, Stenzel-Poore MP. The use of flow cytometry to evaluate temporal changes in inflammatory cells following focal cerebral ischemia in mice. Brain Res. 2002;932:110-9.

28. Larochelle C, Alvarez JI, Prat A. How do immune cells overcome the bloodbrain barrier in multiple sclerosis? FEBS Lett. 2011;585:3770-80.

29. Prat A, Biernacki K, Lavoie JF, Poirier J, Duquette P, Antel JP. Migration of multiple sclerosis lymphocytes through brain endothelium. Arch Neurol. 2002;59:391-7.

30. Luissint AC, Artus C, Glacial F, Ganeshamoorthy K, Couraud PO. Tight junctions at the blood brain barrier: physiological architecture and diseaseassociated dysregulation. Fluids Barriers CNS. 2012;9:23.

31. Persidsky $Y$. Insights into end-organ injury in HIV infection: dynamics of monocyte trafficking to the brain in SIV encephalitis. Am J Pathol. 2015;185: 1548-51.

32. Tobin MK, Bonds JA, Minshall RD, Pelligrino DA, Testai FD, Lazarov O. Neurogenesis and inflammation after ischemic stroke: what is known and where we go from here. J Cereb Blood Flow Metab. 2014;34:1573-84.

33. Fu Y, Liu Q, Anrather J, Shi FD. Immune interventions in stroke. Nat Rev Neurol. 2015;11:524-35.

34. Chamorro A, Hallenbeck J. The harms and benefits of inflammatory and immune responses in vascular disease. Stroke. 2006;37:291-3.

35. Rom S, Zuluaga-Ramirez V, Dykstra H, Reichenbach N, Ramirez SH, Persidsky Y. Poly (ADP-ribose) polymerase-1 inhibition in brain endothelium protects the blood-brain barrier under physiologic and neuroinflammatory conditions. J Cereb Blood Flow Metab. 2015;35:28-36.

36. Ramirez SH, Heilman D, Morsey B, Potula R, Haorah J, Persidsky Y. Activation of peroxisome proliferator-activated receptor gamma (PPARgamma) suppresses Rho GTPases in human brain microvascular endothelial cells and inhibits adhesion and transendothelial migration of HIV-1 infected monocytes. J Immunol. 2008;180:1854-65

37. Bernas MJ, Cardoso FL, Daley SK, Weinand ME, Campos AR, Ferreira AJ, Hoying JB, Witte MH, Brites D, Persidsky Y, et al. Establishment of primary cultures of human brain microvascular endothelial cells to provide an in vitro cellular model of the blood-brain barrier. Nat Protoc. 2010:5:1265-72

38. Aoyagi-Scharber M, Gardberg AS, Yip BK, Wang B, Shen Y, Fitzpatrick PA. Structural basis for the inhibition of poly (ADP-ribose) polymerases 1 and 2 by BMN 673, a potent inhibitor derived from dihydropyridophthalazinone. Acta Crystallogr F Struct Biol Commun. 2014;70:1143-9.

39. Rom S, Reichenbach NL, Dykstra H, Persidsky Y. The dual action of poly (ADP-ribose) polymerase -1 (PARP-1) inhibition in HIV-1 infection: HIV-1 LTR inhibition and diminution in Rho GTPase activity. Front Microbiol. 2015;6:878.

40. Mukhopadhyay P, Rajesh M, Cao Z, Horvath B, Park O, Wang H, Erdelyi K, Holovac E, Wang Y, Liaudet L, et al. Poly (ADP-ribose) polymerase-1 is a key mediator of liver inflammation and fibrosis. Hepatology. 2014;59:1998-2009.

41. Ramirez SH, Hasko J, Skuba A, Fan S, Dykstra H, McCormick R, Reichenbach N, Krizbai I, Mahadevan A, Zhang M, et al. Activation of cannabinoid receptor 2 attenuates leukocyte-endothelial cell interactions and bloodbrain barrier dysfunction under inflammatory conditions. J Neurosciences. 2012;32:4004-16. 
42. Zuluaga-Ramirez V, Rom S, Persidsky Y. Craniula: A cranial window technique for prolonged imaging of brain surface vasculature with simultaneous adjacent intracerebral injection. Fluids Barriers CNS. 2015;12:24

43. Rom S, Dykstra H, Zuluaga-Ramirez V, Reichenbach NL, Persidsky Y. miR-98 and let- $7 \mathrm{~g}^{*}$ protect the blood-brain barrier under neuroinflammatory conditions. J Cereb Blood Flow Metab. 2015;35:1957-65.

44. Goldey GJ, Roumis DK, Glickfeld LL, Kerlin AM, Reid RC, Bonin V, Schafer DP, Andermann ML. Removable cranial windows for long-term imaging in awake mice. Nat Protoc. 2014;9:2515-38

45. Lescot T, Fulla-Oller L, Palmier B, Po C, Beziaud T, Puybasset L, Plotkine M, Gillet B, Meric P, Marchand-Leroux C. Effect of acute poly (ADP-ribose) polymerase inhibition by $3-\mathrm{AB}$ on blood-brain barrier permeability and edema formation after focal traumatic brain injury in rats. J Neurotrauma. 2010;27:1069-79.

46. Moroni F. Poly (ADP-ribose) polymerase 1 (PARP-1) and postischemic brain damage. Curr Opin Pharmacol. 2008;8:96-103.

47. Rouleau M, Patel A, Hendzel MJ, Kaufmann SH, Poirier GG. PARP inhibition: PARP1 and beyond. Nat Rev Cancer. 2010;10:293-301.

48. Veres B, Gallyas Jr F, Varbiro G, Berente Z, Osz E, Szekeres G, Szabo C, Sumegi B. Decrease of the inflammatory response and induction of the Akt/ protein kinase $B$ pathway by poly-(ADP-ribose) polymerase 1 inhibitor in endotoxin-induced septic shock. Biochem Pharmacol. 2003;65:1373-82.

49. Lenzser G, Kis B, Snipes JA, Gaspar T, Sandor P, Komjati K, Szabo C, Busija DW. Contribution of poly (ADP-ribose) polymerase to postischemic blood-brain barrier damage in rats. J Cereb Blood Flow Metab. 2007;27:1318-26.

50. Farez MF, Quintana FJ, Gandhi R, Izquierdo G, Lucas M, Weiner HL. Toll-like receptor 2 and poly (ADP-ribose) polymerase 1 promote central nervous system neuroinflammation in progressive EAE. Nat Immunol. 2009;10:958-64

51. Jagtap P, Szabo C. Poly (ADP-ribose) polymerase and the therapeutic effects of its inhibitors. Nat Rev Drug Discov. 2005;4:421-40.

52. Zhang C, Yang J, Jennings LK. Attenuation of neointima formation through the inhibition of DNA repair enzyme PARP-1 in balloon-injured rat carotid artery. Am J Physiol Heart Circ Physiol. 2004;287:H659-66.

53. Barthel SR, Johansson MW, McNamee DM, Mosher DF. Roles of integrin activation in eosinophil function and the eosinophilic inflammation of asthma. J Leukoc Biol. 2008;83:1-12.

54. Salas A, Shimaoka M, Kogan AN, Harwood C, Von Andrian UH, Springer TA. Rolling adhesion through an extended conformation of integrin alphaLbeta2 and relation to alpha I and beta I-like domain interaction. Immunity. 2004;20:393-406.

55. Lim YC, Wakelin MW, Henault L, Goetz DJ, Yednock T, Cabanas C, SanchezMadrid F, Lichtman AH, Luscinskas FW. Alpha4beta1-integrin activation is necessary for high-efficiency T-cell subset interactions with VCAM-1 under flow. Microcirculation. 2000;7:201-14.

56. Ferreira AM, Isaacs H, Hayflick JS, Rogers KA, Sandig M. The p110delta isoform of PI3K differentially regulates beta1 and beta2 integrin-mediated monocyte adhesion and spreading and modulates diapedesis. Microcirculation. 2006;13:439-56.

57. Sanchez-Martin L, Sanchez-Sanchez N, Gutierrez-Lopez MD, Rojo Al, VicenteManzanares M, Perez-Alvarez MJ, Sanchez-Mateos P, Bustelo XR, Cuadrado A, Sanchez-Madrid F, et al. Signaling through the leukocyte integrin LFA-1 in T cells induces a transient activation of Rac- 1 that is regulated by Vav and PI3K/Akt-1. J Biol Chem. 2004:279:16194-205.

58. Woska Jr JR, Shih D, Taqueti VR, Hogg N, Kelly TA, Kishimoto TK. A smallmolecule antagonist of LFA-1 blocks a conformational change important for LFA-1 function. J Leukoc Biol. 2001;70:329-34.

59. Bolomini-Vittori M, Montresor A, Giagulli C, Staunton D, Rossi B, Martinello M, Constantin G, Laudanna C. Regulation of conformer-specific activation of the integrin LFA-1 by a chemokine-triggered Rho signaling module. Nat Immunol. 2009;10:185-94.

60. Alevriadou BR. CAMs and Rho small GTPases: gatekeepers for leukocyte transendothelial migration. Focus on "VCAM-1-mediated Rac signaling controls endothelial cell-cell contacts and leukocyte transmigration". Am J Physiol Cell Physiol. 2003;285:C250-2.

61. Rolfe BE, Worth NF, World CJ, Campbell JH, Campbell GR. Rho and vascular disease. Atherosclerosis. 2005;183:1-16.

62. Van Buul JD, Hordijk PL. Signaling in leukocyte transendothelial migration. Arterioscler Thromb Vasc Biol. 2004:24:824-33.
63. Etienne-Manneville S, Manneville JB, Adamson P, Wilbourn B, Greenwood J, Couraud PO. ICAM-1-coupled cytoskeletal rearrangements and transendothelial lymphocyte migration involve intracellular calcium signaling in brain endothelial cell lines. J Immunol. 2000;165:3375-83.

64. Samstag Y, Eibert SM, Klemke M, Wabnitz GH. Actin cytoskeletal dynamics in T lymphocyte activation and migration. J Leukoc Biol. 2003;73:30-48.

65. Vicente-Manzanares M, Sanchez-Madrid F. Role of the cytoskeleton during leukocyte responses. Nat Rev Immunol. 2004;4:110-22.

66. Mizuno K. Signaling mechanisms and functional roles of cofilin phosphorylation and dephosphorylation. Cell Signal. 2013;25:457-69.

67. Kameoka M, Ota K, Tetsuka T, Tanaka Y, Itaya A, Okamoto T, Yoshihara K Evidence for regulation of NF-kappaB by poly (ADP-ribose) polymerase. Biochem J. 2000;346(Pt 3):641-9.

68. Liu L, Ke Y, Jiang X, He F, Pan L, Xu L, Zeng X, Ba X. Lipopolysaccharide activates ERK-PARP-1-RelA pathway and promotes nuclear factor-kappaB transcription in murine macrophages. Hum Immunol. 2012;73:439-47.

69. Nakagawa $Y$, Sedukhina AS, Okamoto N, Nagasawa S, Suzuki N, Ohta T, Hattori $H$, Roche-Molina M, Narvaez AJ, Jeyasekharan AD, et al. NF-kB signaling mediates acquired resistance after PARP inhibition. Oncotarget. 2015;6:3825-39.

70. Vuong B, Hogan-Cann AD, Alano CC, Stevenson M, Chan WY, Anderson CM, Swanson RA, Kauppinen TM. NF-kappaB transcriptional activation by TNFalpha requires phospholipase C, extracellular signal-regulated kinase 2 and poly (ADP-ribose) polymerase-1. J Neuroinflammation. 2015;12:229.

71. Zohrabian VM, Forzani B, Chau Z, Murali R, Jhanwar-Uniyal M. Rho/ROCK and MAPK signaling pathways are involved in glioblastoma cell migration and proliferation. Anticancer Res. 2009;29:119-23.

72. Tormos Ana M, Talens-Visconti R, Jorques M, Perez-Garrido S, BonoraCentelles A, Nebreda Angel R, Sastre J. p38alpha deficiency and oxidative stress cause cytokinesis failure in hepatocytes. Free Radic Biol Med. 2014; 75(1):S19.

73. Ba X, Garg NJ. Signaling mechanism of poly (ADP-ribose) polymerase-1 (PARP-1) in inflammatory diseases. Am J Pathol. 2011;178:946-55.

74. Shimizu Y, Dobashi K, lizuka K, Horie T, Suzuki K, Tukagoshi H, Nakazawa T, Nakazato $Y$, Mori M. Contribution of small gtpase rho and its target protein rock in a murine model of lung fibrosis. Am J Respir Crit Care Med. 2001; 163:210-7.

75. Haider L. Inflammation, iron, energy failure, and oxidative stress in the pathogenesis of multiple sclerosis. Oxid Med Cell Longev. 2015;2015:725370.

76. Uttara B, Singh AV, Zamboni P, Mahajan RT. Oxidative stress and neurodegenerative diseases: a review of upstream and downstream antioxidant therapeutic options. Curr Neuropharmacol. 2009;7:65-74.

77. Milzani A, DalleDonne I, Colombo R. Prolonged oxidative stress on actin. Arch Biochem Biophys. 1997:339:267-74.

78. Bai P, Nagy L, Fodor T, Liaudet L, Pacher P. Poly (ADP-ribose) polymerases as modulators of mitochondrial activity. Trends Endocrinol Metab. 2015;26:75-83.

79. Pacher $P$, Beckman JS, Liaudet L. Nitric oxide and peroxynitrite in health and disease. Physiol Rev. 2007;87:315-424.

80. Mathews MT, Berk BC. PARP-1 inhibition prevents oxidative and nitrosative stress-induced endothelial cell death via transactivation of the VEGF receptor 2. Arterioscler Thromb Vasc Biol. 2008;28:711-7.

81. Venter G, Oerlemans FT, Willemse M, Wijers M, Fransen JA, Wieringa B. NAMPT-mediated salvage synthesis of NAD+ controls morphofunctional changes of macrophages. PLoS One. 2014;9, e97378.

82. Virag $L$, Szabo $C$. The therapeutic potential of poly (ADP-ribose) polymerase inhibitors. Pharmacol Rev. 2002;54:375-429.

83. Hottiger MO. Poly (ADP-ribose) polymerase inhibitor therapeutic effect: are we just scratching the surface? Expert Opin Ther Targets. 2015;19:1149-52.

84. Jin R, Yang G, Li G. Inflammatory mechanisms in ischemic stroke: role of inflammatory cells. J Leukoc Biol. 2010;87:779-89.

85. Del Zoppo GJ. Inflammation and the neurovascular unit in the setting of focal cerebral ischemia. Neuroscience. 2009;158:972-82.

86. Knowland D, Arac A, Sekiguchi KJ, Hsu M, Lutz SE, Perrino J, Steinberg GK, Barres BA, Nimmerjahn A, Agalliu D. Stepwise recruitment of transcellular and paracellular pathways underlies blood-brain barrier breakdown in stroke. Neuron. 2014;82:603-17. 